\title{
Electron Wave Functions in Heteronano- structures Control the Electron Transfer Dynamics
}

\author{
Lars Dworak ${ }^{1}$, Sina Roth $^{1}$ and Josef Wachtveitl ${ }^{1, *}$ \\ ${ }^{1}$ IPTC, Goethe-University, Max-von-Laue-Straße 7, 60438 Frankfurt, Germany
}

\begin{abstract}
Electron transfer dynamics in $\mathrm{CdTe} / \mathrm{CdSe}$ and $\mathrm{CdSe} / \mathrm{CdS}$ core/shell heteronanostructures decorated with molecular acceptors are determined via transient absorption spectroscopy. The CdSe shell accelerates the electron transfer whereas the CdS shell leads to a retardation.
\end{abstract}

\section{Introduction}

The optical properties of semiconductor quantum dots (QD) with large extinction coefficients and strong photoluminescence can be utilized in different application fields such as light emitting diodes[1], lasers[2] and photovoltaics[3]. The properties are not only tuneable by the choice of the material and the particle size, but also by the growth of an inorganic shell. Larger band gap semiconductors as shell material can lead to type-I heteronanostructures (HNS) where the exciton is confined mainly to the core leading to an increased photostability and high photoluminescence quantum yields. On the other hand type-II HNS spatially separate the charge carriers between core and shell. If one of the charge carriers is distributed over the complete HNS this is typically referred to as the quasi type-II regime. The physical properties of the HNS and hence the classification of a HNS critically depends on the chosen material and the core and shell dimensions [4]. Here we choose to different $\mathrm{HNS}, \mathrm{CdTe} / \mathrm{CdSe}$ and $\mathrm{CdSe} / \mathrm{CdS}$, and investigated the optical and charge transfer properties in presence of molecular electron acceptors.

\section{Results and Discussion}

The HNS were synthesized according literature known procedures[5,6]. The particle diameters and shell thicknesses have been determined by transmission electron microscopy (Fig 1 (A) and (B)). In case of CdTe/CdSe the CdTe core size and CdSe shell thickness were $3.2 \mathrm{~nm}$ and $0.7 \mathrm{~nm}$, respectively. The shell thickness corresponds to $\sim 2$ monolayers (ML) of CdSe. The growth of CdSe leads to a drastic shift of the lowest exciton transition by $0.29 \mathrm{eV}$ (Fig 1(A)). However, the excitonic structure is still preserved indicating that the investigated $\mathrm{CdTe} / \mathrm{CdSe} \mathrm{HNS}$ can be classified as quasi type-II. In the case of $\mathrm{CdSe} / \mathrm{CdS}$

\footnotetext{
*Corresponding author: wveitl@theochem.uni-frankfurt.de
} 
large CdSe cores $(6.1 \mathrm{~nm})$ were coated with three different CdS shell thicknesses, 0.5 ML, $1 \mathrm{ML}$ and $1.5 \mathrm{ML}$. Only a minor shift $(12 \mathrm{meV})$ of the CdSe lowest exciton transition is observed for the CdSe/CdS HNS (Fig. 1 (B)), indicating that the electronic structure is hardly affected by the shell growth. We conclude that our $\mathrm{CdSe} / \mathrm{CdS}$ particles can be understood as type-I HNS.

(A) Quasi Type-II
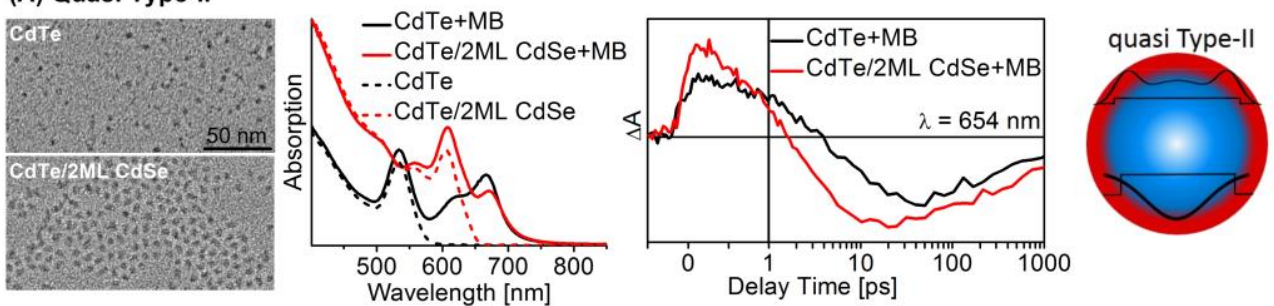

(B) Type-I
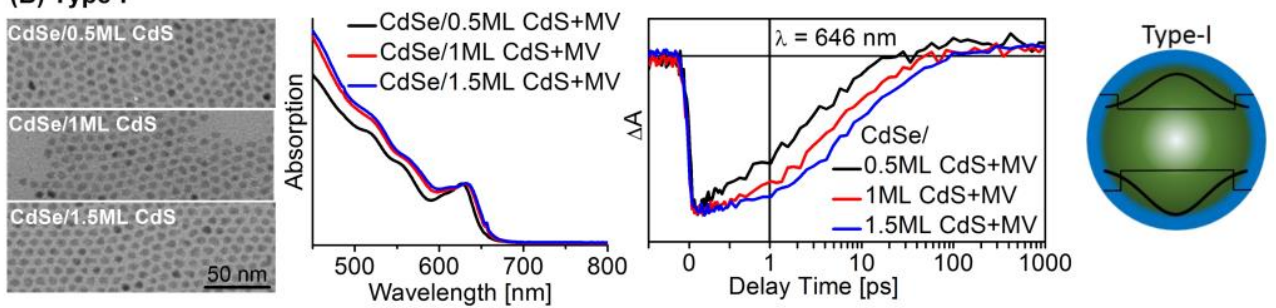

Fig. 1. Transmission electron micrographs of (A) CdTe and CdTe/2ML CdSe, and (B) $\mathrm{CdSe} / \mathrm{CdS}$ with different shell thicknesses. Absorption spectra of (A) CdTe, CdTe/2ML CdSe and the corresponding complexes with $\mathrm{MB}$, and (B) MV-CdSe/CdS complexes (different CdS shell thicknesses). TA traces of (A) MB-CdTe and MB-CdTe/2ML CdSe complexes, and (B) MV$\mathrm{CdSe} / \mathrm{CdS}$ complexes (different $\mathrm{CdS}$ shell thicknesses). Scheme of the electron and hole wave functions of (A) CdTe/CdSe quasi type-II HNS and (B) CdSe/CdS type-I HNS.

For the investigation of the charge transfer properties of the HNS, complexes with two different electron acceptors have been prepared. In case of $\mathrm{CdTe} / \mathrm{CdSe}$, the well-known acceptor methylene blue (MB) was applied. From the absorption spectra of MB-CdTe and MB-CdTe/2ML CdSe complexes MB:HNS molar ratios of 2.2:1 and 2.1:1 are calculated, respectively. The MB exhibits a strong absorption band in the spectral range of $600-750 \mathrm{~nm}$ (Fig 1(A)) which can be used as an indicator for the ET reaction in the transient absorption (TA) measurements. Since the lowest exciton transition of the CdSe/CdS HNS at $\sim 630 \mathrm{~nm}$ strongly overlaps with the $\mathrm{MB}$ absorption band a different electron acceptor (methylviologen, MV) has been chosen. MV is transparent in the visible spectral region, whereas its reduced form exhibits a broad absorption band $(450-750 \mathrm{~nm})$. The MV$\mathrm{CdSe} / \mathrm{CdS} \mathrm{HNS}$ complexes have been prepared at a molar ratio of 10:1. In both cases, the $\mathrm{CdTe} / \mathrm{CdSe}$ and the $\mathrm{CdSe} / \mathrm{CdS} \mathrm{HNS}$, the addition of the molecular acceptor leads to a strong quenching of the HNS photoluminescence (data not shown) indicating an efficient electron transfer (ET) from the photoexcited HNS to the respective molecular acceptor.

To determine the ET dynamics TA measurements have been performed. In the applied setup a noncollinear optical parametric amplifier provides pump pulses at the desired wavelength. A low pump photon flux was used to avoid multiexcitations. The spectrally broad probe pulses are generated by focusing the laser fundamental into a sapphire crystal.

For the selective photoexcitation of the CdTe QD and CdTe/CdSe HNS in the corresponding complexes with $\mathrm{MB}$, the excitation wavelength was set to $490 \mathrm{~nm}$. Transient traces for MB-CdTe and MB-CdTe/2ML CdSe recorded at the spectral position of the MB absorption band $(654 \mathrm{~nm})$ are depicted in Fig. 1(A). For both samples a short-lived positive 
signal turns to a pronounced bleaching which is still observable at maximal delay time. The positive signal stems from the photoexcited QD or HNS and is typically assigned to the carrier-induced Stark effect and intraband hole transitions[7]. The strong bleach at $>1 \mathrm{ps}$ is related to the reduction of the $\mathrm{MB}$ in the ET reaction. Obviously, the formation of this bleach and consequently the ET reaction is faster for the MB-CdTe/2ML CdSe compared to MB-CdTe. To quantify the ET dynamics the half-life times $\left(\tau_{1 / 2}\right)$ are determined from the shown TA traces. The yielded $\tau_{1 / 2}$ are $1.6 \mathrm{ps}$ and $4.1 \mathrm{ps}$ for MB-CdTe/2ML CdSe and MBCdTe, respectively.

The TA traces recorded at $646 \mathrm{~nm}$ after excitation of MV-CdSe/CdS complexes at $630 \mathrm{~nm}$ are depicted in Fig. 1(B). The decay of a pronounced negative signal (HNS lowest exciton bleach) is followed by the formation of a weak positive contribution at each investigated shell thickness. The HNS lowest exciton bleach is predominately evoked by the state filling effect[8] and therefore reflects the population of the $1 \mathrm{~S}(\mathrm{e})$ level of the heterostructure. The weak positive contribution at long delay times indicates the reduction of MV and therefore testifies the expected ET reaction. Comparison of the transient traces shows that the increase of the CdS shell thickness gradually retards the HNS exciton bleach decay dynamics. The $\tau_{1 / 2}$ are 2.3 ps, 4.8 ps and 7.7 ps for the complexes of CdSe/0.5ML $\mathrm{CdS}, \mathrm{CdSe} / 1 \mathrm{ML} \mathrm{CdS}$ and CdSe/1.5ML CdS, respectively.

In summary the CdSe shell strongly affects the CdTe electronic structure (shift of lowest exciton transition) in the $\mathrm{CdTe} / \mathrm{CdSe} \mathrm{HNS}$ and accelerates the ET to MB by a factor of $>2$ compared to the plain CdTe core. From these results we predict a significant probability for the location of the $1 \mathrm{~S}$ electron wave function within the CdSe shell (c.f. scheme in Fig 1(A)) providing an increased donor-acceptor wave function overlap compared to the plain CdTe core. In contrast the CdS shell has only a minor effect on the electronic structure of the CdSe core and retards the electron transfer to MV. This indicates that the electron is confined to the core (c.f. scheme in Fig 1(B)) and the CdS shell acts as an electronic barrier which reduces the donor-acceptor wave function overlap.

\section{References}

1. V.L. Colvin, M.C. Schlamp, A.P. Alivisatos, Nature 370, 354 (1994)

2. V.I. Klimov, A.A. Mikhailovsky, Su Xu, A. Malko, J.A. Hollingsworth, C.A. Leatherdale, H.-J. Eisler, M.G. Bawendi, Science 290, 314 (2000)

3. J. Wang, I. Mora-Seró, Z. Pan, K. Zhao, H. Zhang, Y. Feng, G. Yang, X. Zhong, J. Bisquert, J. Am. Chem. Soc. 135, 15913 (2013)

4. C. de Mello Donegá, Chem. Soc. Rev. 40, 1512 (2011)

5. L. Dworak, S. Roth, M.P. Scheffer, A.S. Frangakis, J. Wachtveitl, Nanoscale, 10, 2162 (2018)

6. L. Dworak, V.V. Matylitsky, V. Breus, M. Braun, T. Basché, J. Wachtveitl, J. Phys. Chem. C 115, 3949 (2011)

7. J. Huang, Z. Huang, S. Jin and T. Lian, J. Phys. Chem. C 112, 19734 (2008)

8. V. Klimov, S. Hunsche, H. Kurz, Phys. Rev. B: Condens. Matter Mater. Phys. 50, 8110 (1994) 